This paper is published in the open archive of Mid Sweden University

DIVA http://miun.diva-portal.org

with permission of the publisher

Citation for the peer-reviewed published paper:

Norgren M, Edlund H. Ion Specific Differences in Salt Induced Precipitation of Kraft Lignin. Nordic Pulp \& Paper Research Journal. 2003;18(4):403-406.

URL to article at publishers site:

http://dx.doi.org/10.3183/NPPRJ-2003-18-04-p400-403 


\section{Ion specific differences in salt induced precipitation of kraft lignin}

Magnus Norgen and Håkan Edlund, Mid Sweden University,Sundsvall, Sweden

Keywords: Kraft lignin, Alkaline solutions, Stability, Precipitation, Ion specificity, Hofmeister, Lyotropic series

SUMMARY: Kraft lignin (KL) precipitation, measured as turbidity, in alkaline solutions $(\mathrm{pH}=10.5)$ at $70{ }^{\circ} \mathrm{C}$ in presence of various salts was investigated. Special concern was devoted to study ion specific effects on KL from monovalent cations and anions belonging to the so-called Hofmeister or lyotropic series. Thus, in the study the stability of KL solutions containing different inorganic and organic anions as well as various quaternary ammonium bromides were tested. In the case of inorganic anions, $\mathrm{Cl}$ and $\mathrm{Br}$ induced a higher degree of $\mathrm{KL}$ precipitation compared to NO, and SCN . The effects of the two tested sodium uronates were found to diverge and, on a short time-scale, galacturonate showed a better KL stabilizing capacity than $\mathrm{C}^{\prime}$. The influence of various tetraalkyl ammonium bromides was compared with the effects of different alkali metal bromides. As the length of the alkyl chain was increased the KL stability was found to be decreasing. However, at equal concentrations, $\left(\mathrm{CH}_{3}\right)_{4} \mathrm{NBr}$ was found to induce a lower degree of KL precipitation than $\mathrm{NaBr}$. From the outcome of the investigation it can be seen that the effects of different cations on KL precipitation follows the mutual order stated by the classical Hofmeister series, whereas the anionic effect has a reversed correspondence.

ADDRESS OF THE AUTHORS: Magnus Norgren,

Magnus.Norgren@mh.se, Håkan Edlund.

Hakan.Edlund $@$ mh.se, Department of Natural and

Environmental Sciences, Chemistry and Fibre Science and

Communication Network, Mid Sweden University, SE-851 70

Sundsvall, Sweden,

In order to produce a chemical pulp of desired properties, it is of great importance to be aware of lignin solubility and stability under process conditions. Indirectly, this is a considerable task in the development and modification of the kraft process (Johansson et al. 1984) and, probably, it will increase in importance as the progress of chemical pulping proceeds. The effects of various ions on the lignin solution behavior are significant during cooking as well as in the washing and bleaching stages (Johansson et al. 1984; Sundin, Hartler 2000; Norgren et al 2001). The impact of multivalent metal ions, for example, magnesium, calcium, barium and aluminum on lignin solutions and pulp washing is described in the literature (Lindstrom 1980; Sundin, Hartler 2000). In general, very little has been presented regarding the specific interactions between lignin and monovalent ions. Indeed, although being of limited industrial relevance, hydrotropic pulping, where high concentrations of various organophilic salts are added to improve the solvent (water) quality, was invented already in the early 1930's (Rydholm 1965). Recently, it was reported that the phase behaviour of kraft lignin solution is differently affected by ions belonging to the so-called Hofmeister series (Norgren et al. 2002). Hofmeister, or lyotropic effects are known to be present in many different macromolecular and colloidal systems (Hofmeister 1888; Napper 1983; Collins, Washabaugh 1984; Piculell, Nilsson 1990; Ninham, Yaminsky 1997; Jonsson et al. 1998). Especially, the effects by anions on negatively charged systems are notable, since the classical electrostatic approaches do not fit at all. In spite of extensive research, the origin of the lyotropic effects is still not completely understood. Among the theories proposed to explain the phenomena are depletion of ions from regions immediately adjacent to the colloids, perturbations of the water structure at interfaces (Napper 1983; Collins, Washabaugh 1984) and extensions of Gibbs adsorption equation (Piculell, Nilsson 1990; Ninham, Yaminsky 1997; Jonsson et al. 1998). In this investigation, ion specific differences in salt induced precipitation of kraft lignin in alkaline solutions are discussed.

\section{Materials and methods}

\section{Chemicals}

A commercial softwood KL (Indulin AT - lot 123H0189, $\mathrm{M},=1060 \mathrm{~g} \mathrm{~mol}^{-1}, M_{\mathrm{w}} / M_{\mathrm{n}}=5.2$ determined by SEC) was supplied by Aldrich. All salts used were of analytical grade and supplied by Riedel-deHaën or Aldrich. Deionised water was used throughout the investigation. Buffer solutions for $\mathrm{pH}$ calibration were supplied by Mallinckrodt Baker.

\section{Turbidity measurements}

Precipitation of KL was monitored by turbidity measurements. The turbidity was determined using a Hach RATIO/XR 43900 turbidimeter, equipped with a tungsten lamp and operating at all the wavelengths of visible light. All measurements were performed directly on the warm sample cuvettes. The difference in turbidity between a newly heated, still hot sample and the equivalent sample, first heated and chilled, then stored at room temperature for several weeks, was found to be below $10 \%$.

\section{pH-measurements}

A Mettler Delta $340 \mathrm{pH}$-meter equipped with an Ingold Inlab ${ }^{\mathrm{TM}} 404$ electrode with automatic temperature correction was used. The electrode was calibrated against fresh buffer solutions at $\mathrm{pH}=7.00$ and 12.01. All samples were $\mathrm{pH}$-adjusted to an initial $\mathrm{pH}$-value of $10.52 \pm 0.02$. 


\section{Results and discussion}

Lyotropic effects are present in various macromolar and colloidal systems. In Figs. la-b, the influence of different cations and anions on KL system stability followed by turbidity measurements is shown. From Fig. $l a$, it is observed that among the tested cations, the lowest stability is obtained in the presence of cesium ions. Within the series of tested metal ion chlorides, the range in causing precipitation is found to be $\mathrm{Cs}^{+}>\mathrm{Na}^{+}>\mathrm{K}$. Concerning the anions, the lowest KL stability is obtained for $\mathrm{Cl}$, followed by $\mathrm{Br}$ and $\mathrm{NO}_{\rightarrow}^{-}$respectively.

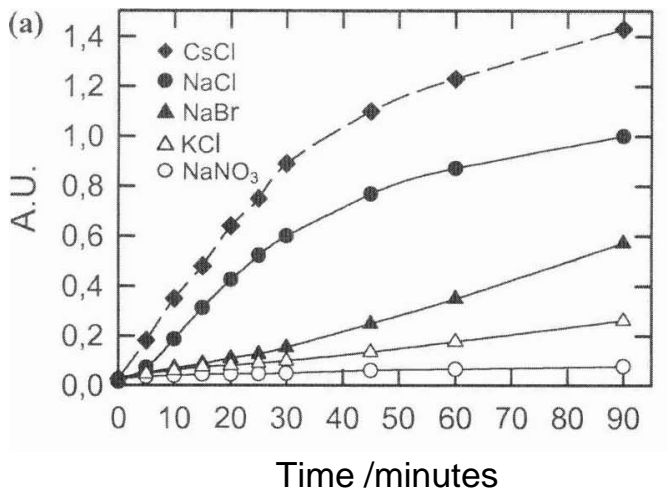

The simplest ion, $\mathrm{NH}_{4}^{+}$, was not tested due to possible $\mathrm{NH}$, formation at the present alkalinities. Figs. $2 a-h$, show the results of the comparison between sodium bromide and some quaternary ammonium bromides. The results presented in Fig. $2 a$ show that tetramethyl ammonium ions are more efficient KL stabilizers than sodium ions. Furthermore, from Fig. $2 b$ it can be observed that compared to the case of sodium ions, the KL solution becomes increasingly more stable at increasing concentration of tetramethyl ammonium ions in samples having a constant background concentration of sodium chloride

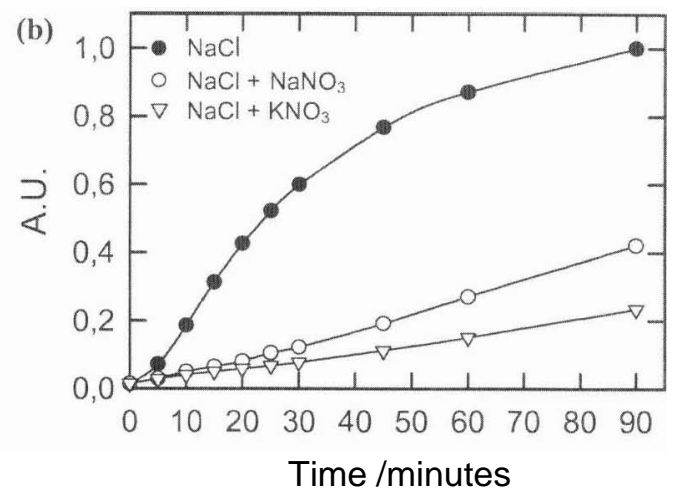

Fig. 1. The figures show the effect of various monovalent metal ion salts on the stability of $\mathrm{KL}$ as monitored by turbidity measurements versus time. (a) The effect of various salts in the Hofrneister series is shown. In (b), the additive effect of anions and cations is demonstrated. Note: All turbidity values are normalized against the end-turbidity of the $\mathrm{NaCl}$ sample in each figure and plotted in Arbitrary Units.

The range of cations in precipitating KL presented in Fig. la, show a reasonable correspondence to the Hofmeister series (Hofmeister 1888; Napper 1983; Collins, Washabaugh 1984). The potency of these cations also follows the sequence for decreasing power in destroying the so-called water structure, which has been determined by IR-measurements on aqueous electrolyte solutions (Napper 1983). According to this, a decrease in KL precipitation could be caused by water structure breaking cations i.e. $\mathrm{K}^{\prime}>\mathrm{Na}^{+}>\mathrm{Li}^{+}>\mathrm{Cs}^{+}$. As the Hofmeister series of monovalent cations proposes a higher macromolecular stability/solubility for some ammonium ions than for alkali metal ions, this was investigated in the KL system.

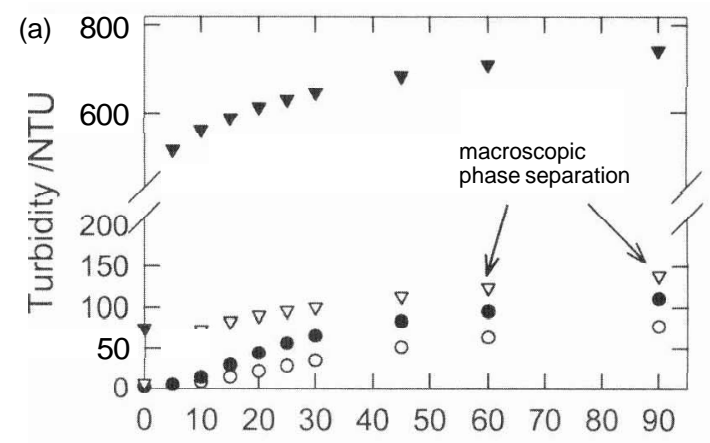

Time /minutes of $0.75 \mathrm{M}$. In the context of chemical pulping this might be a notable observation, since the QUATAM-process, which is based on tetramethyl ammonium hydroxide instead of sodium hydroxide, shows a higher rate of delignification than the kraft process at otherwise equivalent process conditions (Tanczos 1999).

For the investigated anions, on the other hand the tests show an inversed correlation to the classical classification according to Hofmeister. As shown in Figs. la-b and Fig. $3 a, \mathrm{NO}$, and $S C N$ are the best and $\mathrm{Cl}$ is the worst among the tested inorganic anions in providing stability for the alkaline KL system. Interestingly, this coincides with the trend of decreasing surface tension at air-water

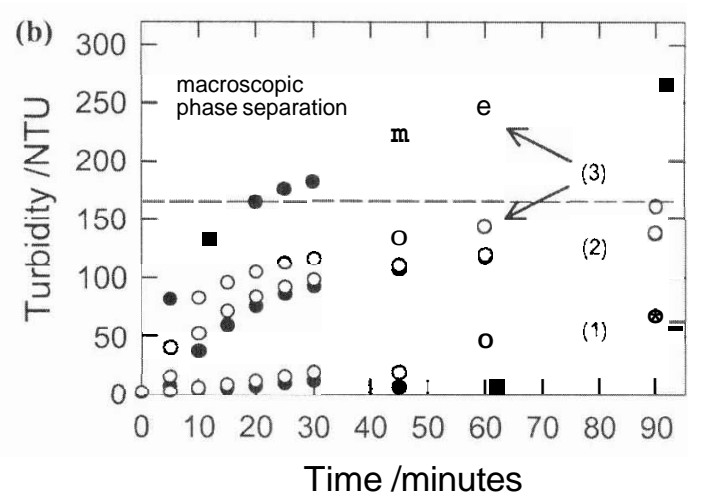

Fig. 2. The figures show the effect of some bromide salts on the stability of $\mathrm{KL}$ at $\mathrm{T}=70{ }^{\circ} \mathrm{C}$ as monitored by turbidity measurements versus time. (a) The effect of various alkyl ammonium bromides $\mathrm{XBr}\left(0 \mathrm{~N}\left(\mathrm{CH}_{3}\right)_{4} \mathrm{Br}, \quad \nabla \mathrm{N}\left(\mathrm{C}_{2}, \mathrm{H}_{4}\right)_{4} \mathrm{Br}, \nabla \mathrm{N}\left(\mathrm{C}_{4} \mathrm{H}_{3}\right)_{4} \mathrm{Br}\right)$ and sodium bromide $(\bullet),[X B r]=0.50 \mathrm{M}$, $[\mathrm{NaCl}]=0.50 \mathrm{M}$ in all the samples. (b) A comparison of $\mathrm{N}\left(\mathrm{CH}_{4}\right)_{4} \mathrm{Br}$ (o) and $\mathrm{NaBr}$ (e) at different concentrations, (1) $0.10 \mathrm{M}$, (2) $0.30 \mathrm{M}$, (3) $0.50 \mathrm{M}$ and a constant background concentration of $0.75 \mathrm{M} \mathrm{NaCl}$ in all the samples. 
interface, where $\mathrm{SCN}$ shows the lowest value and $\mathrm{CO}_{3}{ }^{2}$ and SO,' the highest (Collins, Washabaugh 1984). Furthermore, SCN and NO, are considered very potent water structure breakers (chaotropes), whereas, for instance $\mathrm{SO}$,? belongs to the group of polar water-structure makers (kosmotropes). Moreover, Napper has shown that for poly(oxyethylene) stabilizing moieties, $\mathrm{SO}_{4}^{2-}$ is the most effective anionic flocculant (Napper 1983). Similar solution behaviour regarding various oxyethylene-containing polymers has been observed by others (Piculell, Nilsson 1990; Jonsson et al.). In the latter case, the phenomenon is explained to be due to adsorption of, for instance SCN , on the non-ionic polymer chain. This would create a pseudo-polyelectrolyte and enhanced solubility.

Lyotropic effects are often found to be additive among anions and cations (Napper 1983; Collins, Washabaugh 1984). Fig. Ib shows the aggregation behaviour after addition of sodium chloride and alkali metal ion nitrates to samples containing a fixed standard sodium chloride concentration. As can be seen in Fig. Ib, when sodium chloride is the only electrolyte, extensive aggregation occurs over time. In samples containing a mixture of both $0.375 \mathrm{M} \mathrm{NaCl}$ and $0.375 \mathrm{M} \mathrm{NaNO}_{3}$ or $0.375 \mathrm{M} \mathrm{KNO}$, the aggregation is much less pronounced. The results of the salt test clearly indicates that the effects of cations and anions are additive in the case of KL precipitation. uronates were not analysed for the presence of impurities such as lactones. If lactones are present in the samples, theses species could hydrolyse slowly to their free corresponding acids and thus lead to a slow $\mathrm{pH}$ decrease in the solution. As can be seen in Fig. 3a, glucuronate gives the overall lowest KL stability of all the tested anions. Compared with the studied inorganic ions, the effect of glucuronate ions on KL stability shows a marked time dependency. Initially, the presence of galacturonate induces less significant KL precipitation than do $\mathrm{C} 1$ and $\mathrm{Br}^{-}$. At above 30 minutes, the slope of a thought turbidity curve representing the KL-galacturonate sample rises rapidly and after a while the turbidity of the KL-galacturonate sample exceeds the turbidity of the $\mathrm{Cl}^{-}$and $\mathrm{Br}$ samples. However, as mentioned earlier the comparison between the various anions is not straightforward due the possibilities of further reactions of the uronates or possible impurities with a subsequent decrease in alkalinity, which certainly promotes KL precipitation. The final $\mathrm{pH}$ value of the samples in Fig. $3 a$ was 10.2 for the KL-inorganic salt samples, whereas the $\mathrm{pH}$-values were 9.0 and 8.7 , respectively for the KL-galacturonate and the KLglucuronate samples. This indicates that there are chemical reactions involved responsible for the decrease in $\mathrm{pH}$. In an attempt to distinguish between the different effects of $\mathrm{pH}$ and ion specificity, the $\mathrm{pH}$-values in the uronate samples were measured after different heating times.
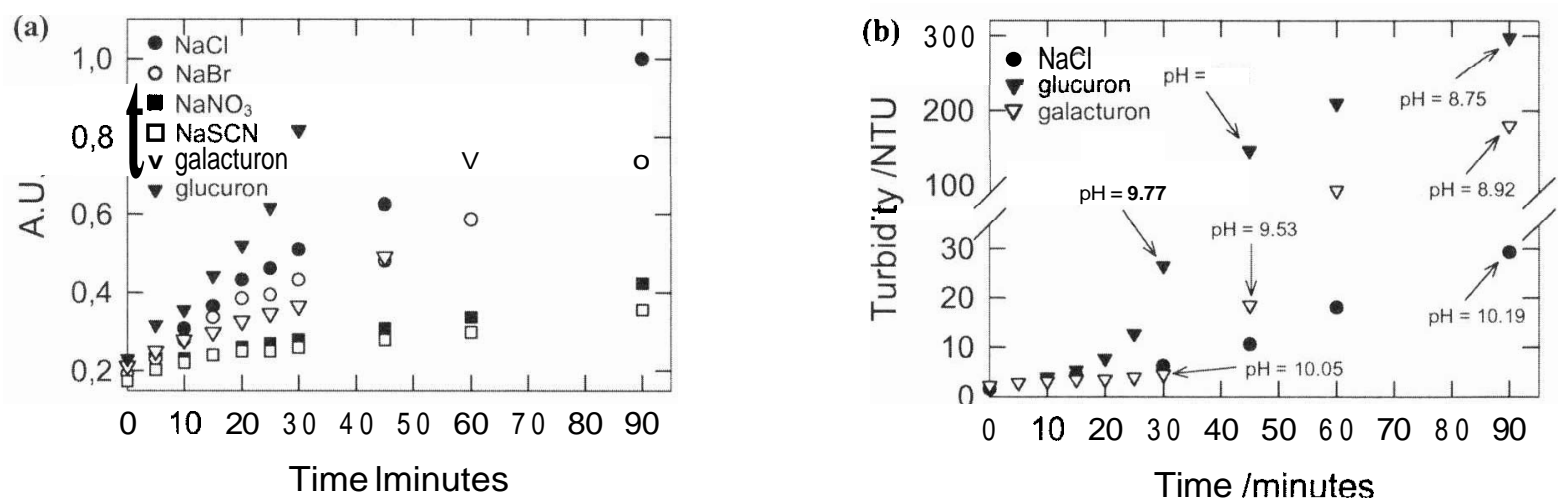

Fig. 3. (a) The effect of various anions on the stability of $\mathrm{KL}$ at $\mathrm{T}=70 \mathrm{C} \mathrm{C}$, as monitored by turbidity measurements versus time. The sodium chloride concentration was $0.375 \mathrm{M}$ in all the samples and the specific salt was added to a concentration of $0.375 \mathrm{M}$. The turbidity values are normalized against the end-turbidity of the $\mathrm{NaCl}$ sample (Arbitrary Units). (b) The effects of sodium chloride and sodium salts of glucuronic and galacturonic acids on the $\mathrm{KL}$ stability. $[\mathrm{NaCl}]=0.800 \mathrm{M}$, [glucuronate, galacturonate] $=0.400 \mathrm{M}+0.400 \mathrm{M} \mathrm{NaCl}$.

Several of the ions tested in this investigation are of course not occurring at important concentrations in ordinary cooking liquors. However, the ion specific interactions between KL and simple ions are of fundamental importance and might provide useful information for the understanding of the stability and solubility of lignin in chemical pulping processes. On the other hand, lyotropic effects are not exclusively caused by inorganic ions (Hofmeister 1888; Napper 1983; Collins, Washabaugh 1984; Piculell, Nilsson 1990; Ninham, Yaminsky 1997; Jonsson et al. 1998). Therefore, KL precipitation in the presence of two different organic sodium salts, D-glucuronate and D-galacturonate, was compared with anions in the Hofmeister series. It should be pointed out that the
From the results shown in Fig. $3 b$ it can be observed that the $\mathrm{pH}$-value in the KL-uronate samples decreases rapidly by heating and at the same time the turbidity increases. However, despite lowering the $\mathrm{pH}$-value, galacturonate seems to possess a precipitation decreasing ability that is totally absent in KL-glucuronate samples. At start, there was an initial turbidity of $1.5 \mathrm{NTU}$ for $\mathrm{KL}-\mathrm{Cl}$ and 2.2 NTU for the KL-galacturonate samples. After 15 minutes at 70 "C, the turbidity had increased to 4.3 and $3.4 \mathrm{NTU}$ $(\mathrm{pH}=10.36)$ and after 30 minutes it was 6.2 and 4.5 NTU, respectively. Thereafter, the turbidity of the KLgalacturonate samples increased steadily above a thought trend line of the $\mathrm{KL}-\mathrm{Cl}^{-}$sample, which is mainly due to the decrease in the $\mathrm{pH}$-value. 
From the results of the present investigation the mutual order of the tested cations and anions to precipitate $\mathrm{KL}$ at medium alkalinities $(\mathrm{pH} \approx 10)$ are as follows:

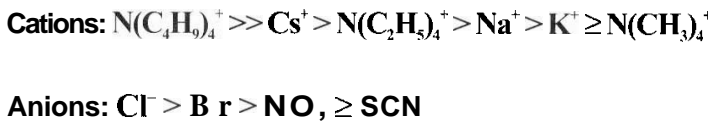

The series of cations given above shows a reasonable match to the original Hofmeister series (Hofmeister 1888). If the earlier described effects of $\mathrm{Ca}^{2}$ and $\mathrm{Mg}^{2+}$ on KL precipitation are included (Sundin, Hartler 2000), this match is further emphasized. From the results of the tested anions on the other hand a completely reversed order compared to the classical Hofmeister series is observed. As can be observed in Figs. 3a-b, concerning glucuronate and galacturonate, one should pay attention to the decrease in the $\mathrm{pH}$-value by time of heating, at least at the investigated alkalinity and temperature. In spite of relatively strong indications of ion specificity the $\mathrm{pH}$ effect induce some uncertainty. Therefore, these ions are not included in the series for anions presented above.

\section{Conclusions}

Ion specific interactions are present in macromolecular systems, alkaline KL solutions should not be excluded. An exact clarification of the phenomenon is not provided in the literature. One explanation suggests a different adsorption/desorption of certain ions at macromolecular interfaces. Another theory emphasises the differences in solution behaviour due to perturbations of the waterstructure by some ions. From the latter school, it is adopted that destabilization of biological macromolecules by salt generally requires a mixture of so-called kosmotropic cations with chaotropic anions. However, in alkaline KL systems the effect seems to be vice versa. More extensive investigations of these ion specific effects are needed. For organic anions this is especially important. For instance, the degradation of hemicelluloses during alkaline cooking creates relatively large concentrations of hydroxy carboxylate residues in the black liquor. Moreover, divalent inorganic anions such as $\mathrm{CO}_{3}{ }^{2}$ and SO,' are also present, but not studied here. When lyotropic effects are shown additive in KL systems, the effect of these anions on KL solubility/stability should be of fundamental interest for further investigations.

\section{Acknowledgements}

The Fibre Science and Communication Network (FSCN) at Mid Sweden University is gratefully acknowledged for financial support. We also thank Referee 1 for valuable comments.

\section{Literature}

Collins, K.D. and Washabaugh, M.W. (1984): The Hofmeister effect and the behaviour of water at interfaces. Q. Rev. Biophys. 18(4):323-422

Hofmeister, F. (1888):Zur Lehre von der Wirkung der Salze. Arch. Exp. Pathol. Pharmakol. 24:247-260.

Johansson, B., Mjoberg, J., Sandstrom, P. and Teder A. (1984): Modified continuous kraft pulping, now a reality. Svensk Papperstidn. 87(10):30-35 (1984).

Jonsson, B., Lindman, B., Holmberg, K. and Kronberg, B. (1998): Surfactants and polymers in aqueous solution, John Wiley \& Sons Ltd, Chichester.

Lindstrom, T. (1980): The colloidal behaviour of kraft lignin. Part II Coagulation of kraft lignin sols in the presence of simple and complex metal ions. Colloid Polymer Sci. 258(2):168-173.

Napper, D.H. (1983): Polymeric stabilization of colloidal dispersions, Academic Press Inc, London.

Ninham, B.W. and Yaminsky, V. (1997): Ion binding and ion specificity: The Hofmeister effect and Onsager and Lifshitz theories. Langmuir 13(7):2097-2108

Norgren, M., Edlund, H., WIgberg, L., Lindstrom, B. and Annergren, G. (2001): Aggregation of kraft lignin derivatives under conditions relevant to the process, part I: phase behaviour. Colloids Surf. A 194(1-3):85-96.

Norgren, M., Edlund, H. and WIgberg, L. (2002): Aggregation of lignin derivatives under alkaline conditions. Kinetics and aggregate structure. Langmuir 18(7):2859-2865.

Piculell, L. and Nilsson, S. (1990): Effects of salts on association and conformational equilibria of macromolecules in solution. Prog. Colloid Polymer Sci. 82:198-210

Rydholm, S.A. (1965): Pulping processes, Wiley-Interscience, New York.

Sundin, J. and Hartler, N. (2000): Precipitation of kraft lignin by metal cations during pulp washing, Nordic Pulp Paper Res. J. 15(4):313-318

Tanczos, I., Putz, R. and Borsa, J. (1999): Comparative study on the effects and mechanism of the new QUATAM-pulping. Proc. 10th ISWPC, June 7-10, Yokohama, Vol. II, pp. 288-291.

Manuscriptreceived October 23,2002 AcceptedMay, 2003 